Mustapha MG
Ashir GM
Rabasa AI
Farouk AG
Elechi HA
Alhaji MA

\title{
Childhood tuberculosis: Characteristics and peculiarities
}

DOI:http://dx.doi.org/10.4314/njp.v47i3.1

Accepted: 17th April 2020

Mustapha MG (匹)

Ashir GM, Rabasa AI, Farouk AG

Elechi HA, Alhaji MA

Department of Paediatrics,

University of Maiduguri

Email: mgofama@gmail.com

\begin{abstract}
Tuberculosis (TB) is an important infectious disease of public health concern. The effects of TB on children in terms of morbidity and mortality is more than that of adults. These may not be unconnected with the differences in characteristics and peculiarities of the disease in children. Tuberculosis in children is usually primary, paucibacillary, characterized by difficulties in diagnosis of both drug susceptible and resistant TB associated with poor uptake of preventive therapy for those with latent infection, contacts and people living with HIV and AIDS (PLWHA) in most developing countries. While significant similarities exist between spectrum of TB occurring in adults and children, the characteristics and peculiarities of childhood TB may be unknown to policy makers and
\end{abstract}

some clinicians, hence giving it less focus in control measures. The aim of this review is to highlight some of the characteristics and peculiarities of TB in children using PubMed/PubMed Central (PMC) and MEDLINE databases searched for relevant search strings from December, 2018 to September, 2019. Appropriate hard copies of books and journal articles were also included.

Conclusion: While the management of TB in children is characterized by difficulties due to both peculiarities of children and the disease; with detailed clinical assessment and examination of necessary specimen in addition to strict adherence to the guidelines of the national TB control program, more cases of TB in children can be prevented, diagnosed, treated and reported.

\section{Introduction}

Tuberculosis (TB) is an important infectious disease of man caused by Mycobacterium tuberculosis, other species that may be pathogenic to humans are $M$ bovis and africanum. Mycobacteria are acid-fast, aerobic, nonspore-forming, non-motile, slow-growing bacilli.

Since the discovery of the bacilli causing the disease in 1882 by Dr Robert Koch, many efforts are put in place to control the disease at both global and National levels. These measures, though being implemented reasonably are yet to bring TB under control especially in the developing countries. Although, both adults and children are infected by the TB bacilli, most of the devastating effects including mortalities are commoner in children and yet control measures for TB are mainly targeted at adults, perhaps due to the epidemiology of the disease. ${ }^{1}$ Since the declaration of TB as a global health emergency and subsequently a number one killer among infectious diseases by the World Health Organization (WHO) in 1993 and 2015, respectively; it is a fact that the disease is far from being controlled particularly in the developing countries. ${ }^{2,3}$
The incidence of TB in Nigeria was put at 338/100,000 and 418,000 for 2012 and 2017, respectively, making Nigeria one of the countries with high burden of TB in the world. ${ }^{4,5}$ Apart from drug sensitive TB (DS-TB); drug resistant TB (DR-TB) and HIV and AIDS are also prevalent in Nigeria. Infection with HIV is the single most important risk for developing TB. The morbidity and mortality due to TB in patients with HIV is also enormous. $^{6,7}$

Drug resistant TB was usually difficult to diagnose even in adults until the GeneXpert MTBRIF test was made available. It is still important to acknowledge that the Xpert machine is not available at all DOTS centers, and where it is available, challenges of logistics for its use or obtaining adequate and suitable samples especially in children for the test abound. ${ }^{8}$ Poverty, poor housing, overcrowding especially in the internally displaced people (IDP) and refugee camps also contribute to the high prevalence of TB in developing countries. Another contributor to the devastating effects of TB in children is inadequate health facilities and qualified health care personnel to offer the needed health care to children in these regions. Looking at the estimate of the global inci 
dence of childhood TB for 2014 by the WHO of 1 million; less than 400,000 was notified that year. ${ }^{5}$

While TB is reported to be more common among adults compared to children, it is estimated that; about $15 \%$ of the total TB cases occurs in the pediatric age group, however, in 2011-2013 in Nigeria; only about 6\% was notified. ${ }^{4,9}$ The low notification rate for childhood TB is a global phenomenon. The dynamics of the percentage of childhood TB of the total TB cases and lag in notification; taking into cognizance under-diagnosis and under-reporting of childhood TB is epidemiologically important.

Although significant similarities exist between spectrum of TB occurring in adults and children, there are important differences in the characteristics and peculiarities of TB in children. These characteristics and peculiarities may be partly responsible for the gap created in the control efforts of TB especially in developing countries. Many of these are obscure especially to policy makers and some clinicians. The aim of this review is to highlight some of the characteristics and peculiarities of childhood TB. We searched the literature using the google search engines in the PubMed/PubMed Central (PMC) and MEDLINE databases, from December, 2018 to September, 2019. Relevant search strings "childhood, pediatric/paediatric TB, DR TB, TB prevention, TBHIV co-infection, TB treatment and other relevant key words were used in the search". Appropriate hard copies of books and journals were also identified and included, in addition to other electronic WHO and United Nations documents. Relevant articles, books and monographs were then selected as references for this review.

\section{Epidemiology of Childhood TB}

The occurrence of $\mathrm{TB}$ in children is a pointer to a recent TB infection in the community; thus, lack of adequate control unlike TB infection in the adult population. This is because most of the TB infection in children progress to a disease within a year or two, ${ }^{10,11} \mathrm{~TB}$ disease in adults is mainly due to reactivation of a dormant infection. An adult with active PTB may infect on the average, between 10 and 15 persons every year. ${ }^{4,12}$ Communities and countries recording high rates of $\mathrm{TB}$ in children suggest that active TB transmission abound in these areas. The annual risk of TB infection for children may be as high as $4 \%$ especially in communities with high prevalence of $\mathrm{TB},{ }^{13}$ and the younger the child, the higher the risk of progression of the TB infection to disease. This may not be unconnected with the relatively lower immunity of the younger children compared to the older ones and the adults. Although the drivers of the TB infection such as poverty with its attendant problems, DR-TB, overcrowded living condition among others, cut across all the age groups; disease progression drivers; such as other infections that weaken immune system, malnutrition etc are commoner in the pediatric population. Likewise, severe forms of TB like TB meningitis and miliary $\mathrm{TB}$ are also commoner in the domain of the pediatric population. $^{14,15}$
Tuberculosis was estimated to have caused 1.3 million deaths among HIV-negative people, and additional 300 000 deaths among HIV-positive people in $2017 .{ }^{5}$ Most of the deaths due to TB in children usually occurs among the under-five children. ${ }^{16}$ The reasons for the high mortality among the U-5s may be due to under or delayed diagnosis, difficulties involved in making the diagnosis coupled with usually severe forms of TB in this category of children among other host factors peculiar to the young children. The majority of the global TB deaths occur in the WHO African and South East Asian regions; these two regions account for $85 \%$ of the combined total of TB deaths in HIV-negative and positive people. ${ }^{3}$

Tuberculosis is transmitted through an air borne droplet infection from person to person. Most of the transmission of TB are by adults/adolescents with pulmonary TB (PTB) to other adults or to children. Children can also infect others if they have open TB, however, this is much less common. The risk of TB infection depends on infectiousness of the source case, the duration and intensity of contact, virulence of the organism, and immune status of the child. ${ }^{17}$ Children with PTB are less contagious because TB in children is described as paucibacillary in nature and less likely to have cavitary lesions in lungs; as pulmonary cavitary lesions in adults may have up to 10 million to 1 billion bacilli compared to 100 10,000 organisms in nodular lesions. ${ }^{18}$ Children also have a less forceful cough that may suspend the mycobacteria in the air, have less endobronchial secretions, produce less sputum and swallow sputum even if produced. ${ }^{6,19-21}$ However, children with DR-TB are reported to be relatively older, have severe disease and tend to have higher rates of smear positivity; thus are more likely to transmit $\mathrm{TB}$ than children with DS-TB. ${ }^{22}$ Young children who swallow sputum are also at risk of developing gastrointestinal TB from an infected sputum; thus the higher prevalence of abdominal TB in children. In addition, the "classical" primary progressive PTB in children usually involves the paratracheal group of lymph nodes; thus, presenting with less cough and hence minimal possibility of transmitting the infection. Likewise, the likelihood of transmitting TB by patients with extra pulmonary TB (EPTB) is almost absent, even for the adults, and these forms of TB are commoner in children. Extra pulmonary TB is therefore a "dead end" disease.

Tuberculosis infection also occurs through the ingestion of an infected unpasteurized milk - M. bovis infection. Transmission of TB by $M$ bovis specie through ingestion of unpasteurized milk is not uncommon worldwide, including developed countries. ${ }^{23,24}$

While in developed countries specie details of mycobacteria causing TB is usually identified, the converse is true for most developing countries; where culture is not routinely done. In fact, a condition for doing culture according to TB Control Program in Nigeria currently is when a DR-TB is diagnosed on gene Xpert, otherwise treatment for TB is given without recourse to culture. Thus, it is not possible to give statistics of what 
proportion of TB is caused by each of the species of the mycobacterium complex in most of the developing countries like Nigeria.

\section{Clinical forms of TB in children}

The clinical forms of TB in children are many with different time frame or time table for presentation with symptoms and signs. Some of the forms of TB occurring in children are described in the time table of TB; in 1948 by Wallgren and later by Marais et al in $2005 .^{11,25}$ The incubation period of 3-8 weeks is followed by hypersensitivity reactions such as fever, erythema nodosum, a positive tuberculin skin test (TST) response and formation of the primary complex visible on chest radiograph occurs. ${ }^{6,11,14}$ One to three months after the primary infection is the period of highest risk for the development of disseminated TB. ${ }^{6,11}$ Although, the time between initial infection and clinically apparent TB disease is variable, age at infection, time since primary infection, type of disease manifestation, host immunity are important factors determining progression to disease.

Going by the spectrum of TB illness, all the clinical entities of TB occur in children; although, some occur more commonly than others. In all the forms of TB, it is of utmost importance and a public health responsibility to make every effort to microbiologically diagnose TB if possible, irrespective of the clinical presentation.

\section{Primary Progressive TB of Childhood}

Tuberculosis in children is usually described as primary progressive in nature, because infection with Mycobacterium tuberculosis complex proceeds to development of TB (synonymous to TB disease) without the organisms becoming dormant in tissues and only to get reactivated to cause the disease as in adult type of TB (secondary TB). Primary TB is characterized by primary complex of TB that includes local infection at the portal of entry and the regional lymph nodes that drain the area. ${ }^{6,26}$ The lung is the portal of entry (primary focus) in $>98 \%$ of cases, usually single and transient with or without an overlying pleural reaction. ${ }^{6,26}$

Before acquired immune responses contain disease progression, bacilli may enter the systemic circulation via the regional lymph nodes with occult hematogenous spread. Disseminated TB occurs if the number of circulating bacilli is large and the host cellular immune response is inadequate to contain the infection. More often the number of bacilli is small, leading to clinically unapparent metastatic foci in many organs. ${ }^{6,26}$ Although seeding of organs of the reticuloendothelial system is common, bacterial replication is more likely to occur in organs with conditions that favor their growth, such as the lung apices, brain, kidneys, and bones. ${ }^{6,26}$

However, in congenital TB, an uncommon disease acquired from mothers with genial TB the primary complex is in the liver or in the lung, if infection is acquired via the hematogenous route through the umbilical vein or by aspiration of infected amniotic fluid, respectively. Congenital TB is not common because, one of the clini- cal manifestations of women with genital TB is mainly infertility. Criteria for diagnosis of congenital TB as revised by Cantwell include the infant may have lesions occurring in the first week of life, primary hepatic complex or caseating hepatic granulomas, maternal genital tract or placental TB or exclusion of postnatal transmission by thorough investigation of contacts. ${ }^{27}$ Other revisions to these criteria are available, but are very similar to the Cantwell criteria. Criteria are developed so as to reduce to the barest minimum over diagnosis of the condition. The diagnosis of congenital TB is further made difficult in Nigeria; where the placenta is immediately disposed after delivery. This is in addition to the fact that about $62 \%$ of women deliver outside health facilities in Nigeria, ${ }^{28}$ and thus examination of the placenta is virtually not done.

The outcome of TB infection in children could manifest no clinical disease with positive TST; this is the usual outcome in most infections. Others may have delayed type hypersensitivity reactions to tubercular protein in form of erythema nodosum, phlyctenular conjuctivitis, dactylitis, pleural effusion and Poncet's disease. ${ }^{29-}$ ${ }^{31}$ These and the progressive primary TB disease are commoner in children compared to the adult population. However, the distinction and classification of patients presenting with hypersensitivity reactions and clinical disease such as pleural effusion and arthritis; into effusion or arthritis due to hypersensitivity or TB disease is often very difficult, as many patients with these entities have active TB and also have TB of other sites. ${ }^{32-34}$

Likewise, children with phlyctenular conjunctivitis were found to have TB involving other parts of the body and the conjunctival lesions resolved with anti TB drugs. 35,36 The narrative is the same for children with erythema nodosum. ${ }^{37}$ Although, on the clinical view point, these reactions or lesions may be due to other infectious and noninfectious conditions, it is pertinent therefore to note that, these so called hypersensitivity entities to mycobacterium tubercular protein; when seen are vigorously evaluated for TB and appropriate management instituted; as the morbidity associated with them may be enormous, and their course not necessarily benign.

\section{Diagnosis of $T B$ in children}

Worldwide the diagnosis of TB especially in children is either bacteriologically confirmed or clinically diagnosed. Due to paucibacillary nature of TB infection in children; isolating the organism in clinical specimen is often difficult. However, clinical diagnosis, especially if "loosely" applied has its own problem of over diagnosis of TB in children as the mimickers are many. To overcome this problem of over diagnosis of TB in children, most countries or TB control programs have developed algorithms for the diagnosis of TB in children. All these algorithms follow the clinical method of taking history, examination and investigations. There may also be additional initial antibiotic treatment, especially for pneumonia. These measures are put in place because the 
microbiological diagnosis of TB is challenging; due to low mycobacterial yield from clinical specimen of children. Although employing the use of algorithms may reduce the problem of over diagnosis, sometimes; it is time consuming and allows for further disease progression. Disease progression particularly in children may be associated with increased morbidity and mortality especially in the younger children. ${ }^{16,38}$ Therefore, if the clinical suspicion of TB is very high and the child is ill, early commencement of treatment for $\mathrm{TB}$ without delay is ideal to reduce the morbidity and mortality associated with TB.

The clinical presentation of TB depends on the site/s of disease involvement. In children multiple site involvement is not uncommon. Common presentation of children with TB include fever, cough, weight loss or poor weight gain, delay/loss of developmental milestones and night sweat. ${ }^{6,39}$ Others are lymph node swellings, seizures, coma in addition to constitutional symptoms. Therefore, the differential diagnoses may include pneumonias, encephalopathies from meningitis or encephalitis, HIV/AIDS, malnutrition and sometimes malignancies.

The most important history to elicit is that of contact with an adult with PTB or chronically coughing adult, if a specific history of PTB cannot be ascertained in the contact. The history of contact may be available but it has to be probed well before it is usually obtained. This history is critical as it will guide the physician on the possibility of drug resistance in the contact of the child; as pediatric TB may be difficult to prove bacteriologically and thus the drug resistance pattern. In the displaced persons and children; history of contact is usually more difficult to obtain as these people change place of abode severally, sometimes for long duration before they reach their destination. Another important mode of presentation of TB that is missed by physicians is when TB presents with fever of unknown origin or pyrexia of unknown origin. There may be no symptom or sign referable to any organ. Such children may present with prolonged fever only or sometimes present with malnutrition that is not responding to adequate dietary management etc. This type of TB presentation is described by some authors as cryptogenic $\mathrm{TB} .^{31}$ High index of suspicion and a focused investigation may however unmask the diagnosis. It is also important that physicians arrive at diagnosis of TB and start treatment, as there is no more place for "trial anti TB"; where treatment is commenced with the view of completing or stopping the treatment depending on whether the patient gets better or not, respectively.

The gold standard for diagnosis of TB remains the culture of the mycobacterium organism; because not all acid-fast bacilli are M. tuberculosis. A bacteriologically diagnosed TB case refers to a presumptive TB case (formally a TB suspect) from whom Mycobacterium tuberculosis is identified from a clinical specimen, either by smear microscopy, culture or WHO-approved rapid diagnostics (WRD) such as GeneXpert MTB/RIF.
Only $<40 \%$ of cases of childhood TB are proven as such, 21,40 and the rate may be $<20 \%$ if gastric aspirate or induced sputum is used. ${ }^{41}$ In some developing countries, the rates may be far less. Although bacteriological diagnosis of TB can be made with microscopy for acid fast bacilli, molecular rapid tests or culture methods, it is currently recommended that all patients being evaluated for TB in Nigeria have specimen analyzed with gene Xpert MTB/RIF. ${ }^{42,43}$ The shortcomings associated with relying solely on this diagnostic test are numerous, especially in pediatric practice in developing countries like Nigeria. These include non-availability of the equipment in all DOTS centers, lack of adequate manpower in all the designated sites, non-availability of convenient samples (quality and quantity) for the tests. Other ancillary investigations in diagnosis of TB especially in children, even though most of them have limited diagnostic values as far as confirmation of the diagnosis is concerned are radiological investigations, tuberculin skin tests (TST), immunological assays, histological examination of tissues etc.

Relevant radiographs of chest, spine or brain neuro images are very essential in diagnosis of TB especially in children where adequate and appropriate samples may not be available. Although a normal chest radiograph does not exclude PTB, some common radiological findings in keeping with PTB in children includehilar lymphadenopathy, bronchopneumonic consolidation, reticulonodular opacities, military opacities and pleural fluid collections. ${ }^{44,45}$ Cavitary lesions in the chest radiograph are not common in children but common in adults or in patients with secondary TB. Sometimes when there is lung paranchymal destruction resulting in liquefaction and subsequent drainage, a cavity may be formed even in the primary PTB. Likewise, calcification on the chest radiograph is also not common in pediatric PTB. Major drawback of radiograph is their lack of specificities in the diagnosis of TB as many other disease entities give similar radiological appearance. Also, interpretation of chest radiographs may be challenging to many physicians as the chest contains many structures; images of some super imposed on others.

Mantoux test, is also employed in evaluation of children for TB, although the test detects only exposure and not disease; but TB disease does not occur without exposure to TB. Though there are many forms of TST, Mantoux test is the only one recommended in children. ${ }^{6,46}$ The major drawback for the use of Mantoux test in the diagnosis of active TB is its low specificity and sensitivity as a result of the many causes of false positive and false negative reactions. ${ }^{6,46,47}$ False negative results may be more marked in children due to the high prevalence of diseases and conditions that result in that, on the other hand false positive results in children can occur due to BCG vaccination or infection with non-tuberculous mycobacteria. $^{6,46,48,49}$

The definition of a positive Mantoux reaction depends on factors such as age, prevalence of TB in the environment, concurrent infections such as HIV or measles, contact with a person with PTB among others. 
Therefore, it is not correct to define a positive Mantoux test as $10 \mathrm{~mm}$ or greater induration in all circumstances. ${ }^{6,46}$ Diagnostic BCG test or accelerated BCG response is another test that is rarely employed in children with the aim of detecting exposure to mycobacteria like the Mantoux test. The diagnostic BCG test produces induration, vesication and ulceration in less than 72 hours in patients exposed to TB; as oppose to several weeks taken before the formation of ulcers in routine BCG vaccination (in children unexposed to TB). ${ }^{31,50}$

Interferon $\gamma$ Release Assay (IGRA) such as T-SPOT.TB and Quanti FERON-TB are blood tests that detect IFN$\gamma$ generation by the patient's $\mathrm{T}$ cells in response to specific $M$. tuberculosis antigens. The IGRA test is more specific and the antigens are not present on M.bovis, BCG and most non tuberculous mycobacteria (NTEM). ${ }^{6,41}$ Internal controls in IGRA makes it useful in certain category of children where TST have limited value; such as the malnourished and the immunocompromised children. Another advantage of IGRA is that of a single encounter as against a dual encounter in TST. Both however, detect exposure and thus none of them differentiates latent TB infection (LTBI) and disease. Each of the IGRA has one cutoff positive value regardless of the age or other peculiarities of the patient. But others argue that there is lack of evidence for the accuracy of the IFN- $\gamma$ tests for specific populations, including HIV-infected or other immunocompromised patients, children etc. ${ }^{41,51}$ An important drawback of the IGRA is high cost of the test, and thus WHO does not endorse its use in low and middle income countries, even in countries with a high prevalence of tuberculosis. ${ }^{6,52}$ Test for HIV infection; although not part of the investigation for TB infection or disease, almost all authors discussing diagnosis of TB include it in the evaluation of patients for TB. This is because, throughout the history of TB, no condition has impacted and influenced TB like HIV infection. The prevalence of TB among PLWHA is high, as about one-third of HIV-infected persons worldwide are infected with TB and $8 \%$ to $10 \%$ of them develop clinical disease every year. ${ }^{7,53,54}$ Most if not all the diagnostic tools and indeed the entire management of TB is influenced by HIV infection. For example, in PLWHA the bacillary load is less, any duration of cough (current cough) should be evaluated for $\mathrm{TB}$, chest radiograph may be atypical and TST results defining positivity is less. ${ }^{6,42,55-57}$ The likelihood of DRTB, TB recurrences, adverse events with treatment and treatment failure is higher in PLWHA. ${ }^{6,58-60}$

The risk of disseminated BCG disease in HIV infected infants vaccinated with BCG was also reported to be much higher compared to non-infected infants, thus WHO recommendation of not giving BCG to children who are known to be infected with HIV; except in places with high TB prevalence. ${ }^{61,62}$ These and many other factors make HIV test almost mandatory in patients being evaluated for TB. Thus, HIV is one of the factors put forward as a cause of resurgence of TB worldwide; with developing countries more affected.

Latent TB Infection refers to infection with M. tubercu- losis in the absence of symptoms, signs or radiological features of disease usually with a positive TST. Infants with LTBI are reported to have high risk for progression to $\mathrm{TB}$ disease and the greatest risk for progression occurs in the first two years after infection. ${ }^{6,14,42,55}$ Therefore, all children younger than 5 years who are close contacts of an infectious index TB patient should be treated for LTBI, likewise PLWHA. However, for children older than 5 years, the need for such treatment should be individualized. The test or investigation for TB exposure in the form of TST or IGRA is not necessary, likewise, chest radiograph, to confirm LTBI or TB disease respectively in low- and middle-incomecountries before commencement of treatment, but a symptomatic screening in the initial instance. ${ }^{63,64}$

Children placed on treatment for LTBI must be followed up and if they develop TB disease during the treatment further investigations and appropriate treatment should be offered. The treatment of LTBI is usually designated preventive therapy because the aim is to prevent development of symptomatic TB disease, although in the actual sense it is a treatment of latent infection. While a lot of controversies exist in the field of screening and treatment of LTBI, the WHO recommends the treatment with isoniazid or other drug/s usually for 6 months of children $<5$ years who are close contacts of an infectious index TB patient and PLWHA based on evidence for the benefits of such treatment. ${ }^{6,55,64}$ The areas of controversies include among others; duration of treatment and regimen, secondary preventive therapy, fear for inducing drug resistance, inclusion or exclusion of other age groups and screening procedures. ${ }^{65,66}$

Also, children who are contacts of DR-TB have high risk of contracting DR-TB, therefore should be screened and given preventive therapy in form of fluoroquinolone with or without another effective drug for prevention, ${ }^{66-}$ ${ }^{67}$ although some authors argue that such recommendation is early for now. ${ }^{68}$ Despite the WHO recommendations for the preventive therapy, a rather low up-take of the treatment and sometimes poor adherence to the recommendation of the treatment by care givers and physiciansrespectively were reported from many studies. $7,65,69,70$

However, a robust training of physicians attending to adults and children, other healthcare workers and counselors in the area of TB preventive therapy in addition to the provision of funds to support the entire exercise may close the policy-practice gap currently being reported especially in TB endemic regions.

Drug resistant TB is grossly under-detected and under reported in children especially in developing countries; it is estimated that 30,000 children become sick with MDR-TB each year globally, and far less children are appropriately diagnosed and treated..$^{71-75}$ This may be due to the difficulty in making the diagnosis of culture confirmed DR-TB in children as oppose to adults. Nigeria is one of the countries with high DR- TB burden due to the high burden of DS-TB and high HIV prevalence. Other factors responsible for the high burden of TB and HIV also apply to the DR-TB burden. Although the possibility of DR-TB is entertained in any patient being 
evaluated for TB, patients with prior treatment for TB or exposure to a possible source case having drug-resistant organisms, high community prevalence of DR-TB and children not responding to first-line therapy despite adherence; are priority group for DR-TB. ${ }^{55,64}$

Despite the challenges in making the diagnosis of DRTB in children, every effort should be made to get clinical specimen for drug susceptibility testing (DST) so as to guide treatment. This is because clinicians in the field always have challenges as to what category of children to treat for DR-TB due to the challenges involved in diagnosis and lack of a reliable history of contact with a source case with DR-TB as reported by Seddon et al. ${ }^{22}$ The guidance defining confirmed DR-TB as a child with clinical TB disease and sample taken from the child shows DR-TB or clinically diagnosed DR-TB (presumed DR-TB) as child with clinical TB disease and has risk factors for drug resistance, ${ }^{71,76}$ is therefore, apt.

The Sentinel Project for Pediatric Drug-Resistant Tuberculosis reports that only $5 \%$ of the estimated children with DR-TB get appropriately treated, ${ }^{71}$ This is as a result of undue delay in commencing treatment thus increasing the morbidity and mortality in children. Other factors that may be responsible for the abysmal number of children being treated for DR-TB could be the fear regarding the toxicity of the second-line TB medications in children and paucity of practice-based recommendations on the optimal care of children with DR-TB. ${ }^{76-}$ ${ }^{77}$ Issues pertaining to dosing of the drugs and appropriate regimen also pose challenges for treatment of children with DR-TB. ${ }^{64,73,76}$

Monitoring of patients and especially children on DRTB treatment is of paramount importance and mandatory. The areas of this monitoring include response to therapy, drug adverse events and treatment adherence monitoring as proposed by Seddon et al. ${ }^{76}$ The monitoring entails routine clinical, laboratory and sometimes radiological assessment of the patient. Even though, the protocol and procedure for the monitoring of patients including children on DR-TB is well outlined, it may be a daunting challenge especially in resource poor countries and in places where Medicare is mainly achieved through out of pocket expenses. This is because most patients cannot afford the costs of the tests even if the drugs are supplied free of charge to the patient. Expertise for doing some of the assessments and tests may also be lacking especially for young children; for example, audiology, visual acuity testing etc.

However, there is great hope for children with DR-TB despite the challenges as the global focus for the control of TB is now integrating the management of childhood TB in its program as against the past experience of neglecting childhood TB. Inspite of the fears and challenges associated with the management of DR-TB, studies have shown that it is very possible to diagnose and treat more cases of even confirmed DR-TB in children in addition to the presumed DR-TB in children. ${ }^{76}$

Tolerability of second line anti-TB drugs in children has been documented in a study from New York of 20 children treated for MDR-TB (six culture-confirmed), demonstrated good outcomes and minimal toxicity. ${ }^{78}$ Simi- larly, a good outcome of DR-TB management in children compared to some adult literature was reported. ${ }^{22,73}$ In a mainly adult study, EL Hamdouni et al reported a relatively poor outcome compared to the WHO standard in their study of DR-TB treatment outcome comprising patients aged 9.5 to 70 years in Morocco. ${ }^{79}$ The development of newer regimen that are for short duration, devoid of injectable agents is usually tolerated by children better. ${ }^{71,73}$ It is recommended that children with non-severe DR-TB disease and severe DR-TB disease are treated with four and five second line anti-TB drugs for $9-12$ months and 12-18 months, respectively. ${ }^{71}$ Treatment of children with DR-TB beyond 18 months is not recommended.

The options for DR-TB treatment are standardized, empiric, and individualized regimens; the approach is dependent on having access to either reliable DST results for individual patients or population data on the prevalent resistance patterns. ${ }^{55}$ Although this is applicable to treatment of all infections in children as well as adults, the most preferredoption of course is the individualized regimen where DST result of an individual is available and treatment is guided by the sensitivity pattern of the individual patient in question. A robust follow-up and monitoring protocol has been developed by the Sentinel Project for Pediatric Drug-Resistant Tuberculosis for treatment of children with DR-TB. ${ }^{71}$

The provision of care in terms of offering treatment to children for DS-TB or DR-TB is slightly different from that of adults who ethically have autonomy. Majority of adults can take their medications on their own especially during the continuation phase of treatment after proper health education is given. On the other hand, children are given medications by their care givers, who also consent to the treatment on their behalf. However, children whose caregivers have challenges or without an identified caregiver e.g. children in remand homes or orphanages should have another person to serve as a treatment supporter for them. ${ }^{42}$ Family members or any person willing to serve as a treatment supporter takes many responsibilities, including the patient swallowing the drugs in his/her presence among others. ${ }^{42,80,81}$ The treatment supporter provides DOTS services outside the health facility, thus reducing the burden of accessing health facility daily for administration of drugs. This is very important because TB treatment may last 6-18 months depending on drug susceptibility pattern. In addition to the duration that is long, treatment supporter will ensure adherence to treatment, follow up and monitoring of the patient etc. The advantages in terms of success rates of TB treatment through the use of treatment supporter has been reported. ${ }^{82}$

\section{$T B$ control and prevention in children}

The TB control programs at country and regional levels are doing a lot to control TB and prevent TB infection and subsequent development of TB disease. A strategy for TB prevention in children includes BCG vaccination at birth. It is estimated that about 100 million children are vaccinated every year with BCG and it affords 
significant protection against disseminated disease and TB meningitis, however, protection against pulmonary TB is inconsistent. ${ }^{83-85}$

The poor protection conferred by BCG in older children and adults have led to the search for a vaccine that may be efficacious in these population. Even though this has been an area of interest for a long time, an ideal vaccine is yet to be developed against TB. However, WHO reports that twelve vaccine candidates are in various phases of clinical trials. ${ }^{5}$ They include candidates to prevent the development of TB infection, TB disease, and candidates to help improve the outcomes of treatment for TB disease. The WHO has recommended that children diagnosed with HIV infection; whether symptomatic or asymptomatic be exempted from BCG vaccination because of the increased risks of BCG disease in such infants. ${ }^{64,86}$ However, HIV exposed children who have no signs or reported symptoms suggestive of HIV infection and whose HIV status are unknown should be vaccinated with BCG in TB endemic countries. ${ }^{64}$

Children who get infected with $\mathrm{TB}$, either vaccinated with BCG or not, through contact with an infectious TB case or demonstration of infection with a TST or IGRA may be prevented from progressing to TB disease through the implementation of IPT as discussed above. This will reduce the reservoir of future TB cases and infector pool; of the about two billion people infected already worldwide. ${ }^{66}$

Apart from TB infection prevention and progression to disease, TB can also be prevented through avoidance or significant reduction of exposure to an infectious TB case. Infection control guidelines for prevention of TB transmission in a variety of settings published by the WHO contains policies that will reduce TB transmission in health care facilities, congregate settings and house- holds. ${ }^{87}$ This is a very important feat in infection prevention and control since the spread of infection is significant with exposure. ${ }^{12,88}$ Epidemiologically an even more important strategy for TB infection control is to ensure rapid diagnosis and initiation of treatment of persons with TB; especially PTB. This is in addition to the prevention of development of drug resistance and restoration of the health of the patient among other benefits. ${ }^{5,55}$ This is crucial to achieving the End TB Strategy targets set for 2030 and 2035.

In children, the prevention of vertical transmission of HIV infection and early initiation of ART in the infected is shown to drastically improve child survival and reduces the risk of TB disease. ${ }^{5,7,89}$ This is because TB thrives well in the setting where HIV is prevalent or where HIV diagnosis and optimal treatment is deficient.

\section{Conclusion}

Children with TB often have challenges in diagnosis, treatment and monitoring of treatment. This is in addition to the inattention to childhood TB by various governments and international policy makers; except for some efforts and interest in childhood TB in the recent years. Children being vulnerable to infection, progression to disease and complications are important to be thought of in all policies and programs aimed at TB control. The usual lack of bacteriological evidence of infection in children should not be taken as an excuse to exclude them in strategies for TB control, but to try and develop ways to circumvent the challenge. The hope for this is high, as data for children is now being published with respect to TB by the WHO.

\section{References}

1. Du Preez K, Schaaf HS, Dunbar R, Swartz A, Bissell K, Enarson DA, Hessling AC. Incomplete registration and reporting of cultureconfirmed childhood tuberculosis diagnosed in hospital. Public Health Action 2011; 1: 19-24.

2. World Health Organization. TB: a global emergency, WHO report on the TB epidemic (WHO/TB/94.177). Geneva: WHO; 1994 (http:// apps.who.int/ iris/ handle/10665/58749.)

3. World Health Organization. Global Tuberculosis Report 2015. Geneva; 2015.
4. Federal Ministry of Health. Training manual for health care providers on diagnosis and management of childhood tuberculosis in Nigeria, 2015

5. Global tuberculosis report 2018. Geneva: World Health Organization; 2018. License: CC BY-NC-SA 3.0 IGO.

6. Starke JR, Cameron LH. Tuberculosis (Mycobacterium tuberculosis). In: Kliegman RM, St Geme JW, Blum NJ, Shah SS, Tasker RC, Wilson KM, Eds. Nelson Textbook of Pediatrics (vol. 1), 21st Edition, Elsevier, Canada (2020): 1565 1582.
7. Mustapha MG, Garba MA, Rabasa, Farouk AG, Elechi HA, Ibrahim BA, AI et al. An Eleven Year Review of the Management of Paediatric HIV/AIDS: Need for Prevention, Early Diagnosis and Treatment. EC Paediatrics2018; 7.5: 389-399

8. Abubakar I, Zignol M, Falzon D, Raviglione M, Ditiu L, Masham S, et al. Drugresistant tuberculosis: time for visionary political leadership.

Lancet Infect Dis.2013;13 (6):529-39. doi: 10.1016/ S1473-3099(13)70030-6.

9. Murray C J, Styblo K, Rouillon A. TB in developing countries: burden, intervention, and cost. Bull Int Union Tuberc Lung Dis 1990; 65(1): 6-24. 
10. Perez-Velez CM, Marais

BJ. Tuberculosis in children. $N$ Engl J Med 2012; 367: 348361 [PubMed] [Google Scholar],

11. Wallgren A. The time-table of tuberculosis. Tubercle 1948; 29: 245-251 PubMed] [Google Scholar]

12. Global Tuberculosis Control. Epidemiology, Strategy, Financing WHO Report 2009 World Health Organization Google Scholar

13. Shanaube K, Sismanidis C, Ayles H, Beyers N,Schaap A, Lawrence K et al. Annual Risk of Tuberculous Infection Using Different Methods in Communities with a High Prevalence of TB and HIV in Zambia and South Africa. PLoS One. 2009;4(11): e7749. doi: 10.1371/journal.pone.0007749

14. Marais BJ, Gie RP, Schaaf HS,Hesseling AC, Obihara CC, Starke JJ, et al. The natural history of childhood intrathoracic tuberculosis: a critical review of literature from the pre -chemotherapy era. Int J Tuberc Lung Dis.2004;8(4):392-402

15. Comstock GW, Livesay VT, Woolpert SF. The prognosis of a positive tuberculin reaction in childhood and adolescence. Am J Epidemiol 1974;99(2):131138).

16. Dodd P, Yuen C, Sismanidis C,Seddon JA, Jenkins HE. The global burden of tuberculosis mortality in children: a mathematical modelling study. Lancet Glob Health 2017; 5: e898906).

17. Seddon JA, Shingadia D. Epidemiology and disease burden of tuberculosis in children: a global perspective. Infection and Drug Resistance, 2014; 7: 153-165. DOIhttps:// doi.org/10.2147/IDR.S45090).

18. Batra V, Ang JY. Pediatric Tuberculosis. Medscape 2018. Accessed may, 2019medicine.medscape.com

19. Marais BJ, Gie RP, Schaaf HS, Beyers N, Donald PR, Starke JR: Childhood pulmonary tuberculosis: old wisdom and new challenges. Am J Resp Crit Care Med. 2006, 173: 1078-1090. 10.1164/rccm.200511-1809SO
20. Piccini P, Chiappini E, Tortoli E, de Martino M, Galli L. Clinical Peculiarities of tuberculosis.BMC Infectious Diseases 2014;14 (Suppl 1): S4https://doi.org/10.1186/1471 -2334-14-S1-S4

21. Marais B, Hesseling A, Gie R, Schaaf H, Enarson D, Beyers $\mathrm{N}$. The bacteriologic yield in children with intrathoracic tuberculosis. Clin Infect Dis 2006, 42:e69-e71. doi:10.1086/502652.

22. Seddon JA, Hesseling AC, Willemse M, Donald PR, Schaaf HS. Cultureconfirmed multidrug-resistant tuberculosis in children: clinical features, treatment, and outcome, Clin Infect Dis. 2012; 54(2):157-66. doi: $10.1093 / \mathrm{cid} /$ $\operatorname{cir} 772$

23. Shitaye JE, Tsegaye W, Pavlik I. Bovine tuberculosis infection in animal and human populations in Ethiopia: a review. Veterinarni Medicina, 2007; 52 (8): 317-332

24. Akudo L Okeke LA, Fawole O, Muhammad M, Okeke IO, Nguku P, Wasswa et al. Bovine tuberculosis: a retrospective study at Jos abattoir, Plateau State, Nigeria. The Pan African Medical Journal. 2016; 25: 202. doi:10.11604/ pamj.2016.25.202.5669).

25. Marais BJ, Donald PR, Gie RP, Schaaf HS, Beyers N. Diversity of disease manifestations in childhood pulmonary tuberculosis. Ann Trop Paed 2005a;25: 79-86 [PubMed] [Google Scholar).

26. Marais BJand Schaaf HS. Tuberculosis in Children. Cold Spring Harb Perspect Med. 2014; 4(9): a017855.

doi: 10.1101/

cshperspect.a017855

27. Cantwell MF, Shehab ZM, Costello AM, Sands L, Green WF, Ewing, Jr EP, et al. Congenital Tuberculosis, $N$ Engl J Med 1994; 330:1051-1054 DOI: 10.1056/ NEJM199404143301505
28. Adedokun ST and Uthman OA. Women who have not utilized health Service for Delivery in Nigeria: who are they and where do they live? BMC Pregnancy and Childbirth 2019, 19:93 https:// doi.org/10.1186/s12884-0192242-6

29. Ellner JJ, Barnes PF, Wallis RS, Modlin RL. The immunology of tuberculous pleurisy. Semin Respir Infect 1988; 3: 335-342

30. Dall L, Long L, Stanford J. Poncet's disease: tuberculous rheumatism. Rev Inf Dis. 1989;11(1):105-7

31. Shennan DH, Kibel AM. Tuberculosis. In: Stanfield P, Brueton M, Chan M, Parkin M, Waterson T, eds. Diseases of Children in the Subtropics and Tropics. 4th ed. London: Edward Arnold, 1991: 519 . 552

32. Abebe W, Abebe B, Molla K and Alemayehu T. Tuberculous Dactylitis: An Uncommon Presentation of Skeletal Tuberculosis. Ethiop J Health Sci. 2016; 26(3): 301-303. PMID: 27358553

33. Vorster MJ,Allwood BW, Diacon AH, Koegelenberg CFN. Tuberculous pleural effusions: advances and controversies, J Thorac Dis, 2015; 7(6): 981-991. doi: 10.3978/j.issn.20721439.2015.02.18. PMID: 26150911

34. Aktürk H, Soydemir D, Abalı S, Somer A, Yekeler E, Köksalan K, Salman N. Poncet's Disease: Reactive Arthritis Due to Tuberculosis, J Pediatr Inf 2015; 9: 000-000. DOI: 10.5152/ced.2015.1789

35. Lahiri K, Landge A, Gahlowt P, Bhattar A, Rai R. Phlyctenular Conjunctivitis and Tuberculosis. Pediatric Infectious Disease J 2015; 34 (6) 675. doi: 10.1097/ INF.0000000000000628

36. Tomar M, Sharma G, Sood T. Phlyctenular Conjunctivitis and Tuberculosis. Int J Ophthalmol Eye Res., 2016; 4(6): 233-236. 
37. Bjorn-Mortensen K, Ladefoged K, Simonsen J,Michelsen SW, Sørensen HCF, Koch A,et al. Erythema nodosum and the risk of tuberculosis in a high incidence setting. Int J Circumpolar Health, 2016; 75: 10.3402/ ijch.v75.32666. PMID: 27784508.

38. Nelson LJ, Wells CD: Global epidemiology of childhood tuberculosis. Int J Tuberc Lung Dis. 2004, 8: 636-47.

39. Mustapha MG, AI Rabasa, GM Ashir, Usman AB, SY Mohammed. Management of Childhood Tuberculosis: The experience in a Tertiary Health Care Facility in Nigeria. Nigerian Medical Practitioner 2010;57(5 -6):75-8).

40. Zar H, Hanslo D, Apolles P, Swingler G, Hussey G. Induced sputum versus gastric lavage for microbiological confirmation of pulmonary tuberculosis in infants and young children: a prospective study. Lancet, 2005; 365:130134.doi:10.1016/S0140-6736 (05)17702-2.

41. Dunn JJ, Starke JR, Revell PA. Laboratory Diagnosis of Mycobacterium tuberculosis Infection and Disease in Children. $J$ Clin Micribiol, 2016; DOI: 10.1128/JCM.03043-15.

42. Federal Ministry of Health. National Tuberculosis, Leprosy \& Buruli ulcer Management \& Control Guidelines 2015.

43. World Health Organization. Automated real-time nucleic acid amplification technology for rapid and simultaneous detection of tuberculosis and rifampicin resistance: Xpert MTB/RIF assay for the diagnosis of pulmonary and extrapulmonary TB in adults and children Policy update), 2013.

44. Ahidjo A, Mustapha MG, Ashir GM, Alhaji MA, Adedokun T. Chest Radiographic Predictors of HIV/AIDS in Childhood Pulmonary Tuberculosis- an African Study. J Asian Scientific Research 2012; 2(5): 310316.
45. Erinie SA: An appraisal of the radiological features of pulmonary tuberculosis in Ilorin. $\mathrm{Ni}$ ger Postgrad Med J. 2003, 10: 264-9.

46. American Academy of Pediatrics, Committee on Infectious Diseases. Screening of tuberculosis in infants and children. Pediatrics 1994; 93: 131-134.

47. Mustapha MG, GM Ashir, MA Alhaji. Mantoux test reactions among children managed for Tuberculosis in Maiduguri, Nigeria. Scandinavian J infectious Disease. 2011; 43: 15-18.

48. Mustapha MG, GM Ashir, AI Rabasa, GA Farouk. Prevalence of BCG Scar Formation among BCG Vaccinated Apparently Health U-5 children and its Correlation with Mantoux Skin induration in Maiduguri, Nigeria. Nig Med J 2008; 49(4): 84-7

49. Mustapha MG, GM Ashir, AI Rabasa, MS Gimba. Prevalence of Mantoux test positivity among apparently healthy children in Maiduguri, Nigeria. South Afri J Child Health 2009; 3(3): 80-2.

50. Rehman A, Idris M. Comparison of mantoux's test with diagnostic BCG in pediatric patients with pulmonary tuberculosis. J Ayub Med Coll Abottabad 2005; 17:6-8

51. Pai M., Riley L. W., Colford J. M., Jr 2004. Interferon-gamma assays in the immunodiagnosis of tuberculosis: a systematic review. Lancet Infect.

Dis.4:761-776

52. World Health Organization. Use of tuberculosis interferongamma release assays (IGRAs) in low- and middle-income countries: policy statement. Geneva, 2011 (WHO/HTM/ TB/2011.18).

53. Joint United Nations Program on HIV/AIDS (UNAIDS)/ World Health Organization. 2008 report on the global AIDS epidemic. Chapter 2, Status of the global epidemic. http:// data.unaids.org/pub/ GlobalReport/2008/ jc1510_2008_global_report_pp 29_62_en.pdf. Accessed 13 May, 2009
54. Gao J, Zheng P, Fu H. Prevalence of TB/HIV Co-Infection in Countries Except China: A Systematic Review and MetaAnalysis, 2013. PLoS ONE 8 (5): e64915. doi:10.1371/ journal.pone.0064915.

55. TB CARE I. International Standards for Tuberculosis Care, Edition 3. TB CARE I, The Hague, 2014.

56. Moyo S, Verver S, Mahomed H, Hawkridge A, Kibel M, Hatherill M, et al. Age-related tuberculosis incidence and severity in children under 5 years of age in Cape Town, South Africa. Int J Tuberc Lung Dis.2010;14:149-154.

57. Chintu C. Tuberculosis and human immunodeficiency virus co-infection in children: management challenges. Paediatr Respir Rev 2007; 8(2): 142-7.

58. Korenromp EL, Scano F, Williams BG, Dye C, Nunn P. Effect of human immunodeficiency virus infection on recurrences of tuberculosis after rifampin-based treatment: an analytical review, Clin Infect Dis, 2003, vol. 37: 101-112

59. Nahid P, Gonzalez LC, Rudoy I, de Jong BC, Unger A, Kawamura LM. et al. Treatment outcomes of patients with HIV and tuberculosis, Am J Respir Crit Care Med, 2007, vol. 175: 1199 1206.

60. Swaminathan S, Padmapriyadarsini C, Narendran G. HIV-Associated Tuberculosis: Clinical Update. Clinical Infectious Diseases, 2010; 50 (10): 1377-1386, https:// doi.org/10.1086/652147

61. Hesseling AC, Marais BJ, Gie RP, Schaaf HS, Fine PEM, Godfrey-Faussett $\mathrm{P}$, et al. The risk of disseminated Bacille Calmette-Guerin (BCG) disease in HIV-infected children, Vaccine 2007, 25; 14-18 
62. WHO, International Union Against Tuberculosis and Lung Disease (The Union). Guidance for national tuberculosis and HIV programs on the management of tuberculosis in HIVinfected children: Recommendations for a public health approach. http:// www.theunion.org/index.php/ en/resources/technicalpublications/item/759-guidance -for-national-tuberculosis-andhiv-programmes-on-themanagement-of-tuberculosis-in -hiv-infected-childrenrecommendations-for-a-publichealth-approach), 2010.

63. Kruk A, Gie RP, Schaaf HS, Marais BJ. Symptom-based screening of child tuberculosis contacts: improved feasibility in resource-limited settings. Pediatrics, 2008, 121:16461652

64. WHO Guidance for national tuberculosis programs on the management of tuberculosis in children - 2nd ed. 2014

65. Reuter A, Hughes J, Furin J. Challenges and controversies in childhood tuberculosis, Lancet 2019; 394: 967-78.

66. Schaaf HS, Marais BJ, Carvalho I, Seddon JA. Challenges in childhood tuberculosis. In: Migliori GB, Bothamley G, Duarte R, Rendon A, eds. Tuberculosis (ERS Monograph). Sheffield, European Respiratory Society, 2018; pp. 234-262 [https://

doi.org/10.1183/2312508X.100 21817]

67. Becerra MC, Frank MF, Appleton SC, Joseph JK, Bayona J, Atwood SS, et al. Tuberculosis in children exposed at home to multidrug-resistant tuberculosis. Pediatric Infectious Disease J, 2012, 31:115-119.

68. Huerga H, Sanchez-Padilla E, Melikyan N, Atshemyan H, Hayrapetyan A, Ulumyan A et $a l$. High prevalence of infection and low incidence of disease in child contacts of patients with drug-resistant tuberculosis: a prospective cohort study. Arch Dis Child 2018; 104: 622-28.
69. Szkwarko D, HirschMoverman Y, Du Plessis L,Du Preez K, Carr C, Mandalakas AM. Child contact management in high tuberculosis burden countries: a mixedmethods systematic review. PLoS One 2017; 12: e0182185.

70. Belgaumkar V, Chandanwale A, Valci C, Pardeshi G, Lokhande R, Kadam D,et al. Barriers to screening and isoniazid preventive therapy for child contacts of tuberculosis patients. Int J Tuberc Lung Dis 2018; 22: 1179-87.

71. The Sentinel Project for Pediatric Drug-Resistant Tuberculosis. Management of DrugResistant Tuberculosis in Children: A Field Guide. Boston, USA; 2018, Fourth edition. (http://sentinel-project.org/)

72. Onyedum CC, Alobu I, Ukwaja KN. Prevalence of drugresistant tuberculosis in Nigeria: A systematic review and meta-analysis. 2017, PLoS ONE 12(7): e0180996. https:// doi.org/ 10.1371/ journal.pone.0180996

73. Schaaf HS. Diagnosis and Management of MultidrugResistant Tuberculosis in Children: A Practical Approach. Indian J Pediatr, 2019; 86(8): 717-724. doi: 10.1007/s12098018-02846-8.

74. Dodd PJ, Sismanidis C, Seddon JA. Global burden of drugresistant tuberculosis in children: a mathematical modelling study, Lancet Infect Dis. 2016 Oct;16(10):1193-1201. doi: 10.1016/S1473-3099(16)30132 -3 .

75. Marais BJ, Schaaf HS. Childhood tuberculosis: an emerging and previously neglected problem. Infect Dis Clin North Am 2010; 24: 727-749.).

76. Seddon JA, Furin JJ, Gale M, Hernan Del Castillo Barrientos HDC, Hurtado RM, Amanullah $\mathrm{F}$, et al. Caring for Children with Drug-Resistant Tuberculosis, Practice-based Recommendations. Am J Respir Crit Care Med 2012, 186; (10): 953-964.
77. Furin J, Tommasi M, GarciaPrats AJ. Drug-resistant tuberculosis: will grand promises fail children and adolescents? Lancet Child Adolesc Health 2018; 2: 237-38.

78. Feja K, McNelley E, Tran CS, Burzynski J, Saiman L. Management of pediatric multidrugresistant tuberculosis and latent tuberculosis infections in New York City from 1995 to 2003. Pediatr Infect Dis J 2008;27(10):907-12.

79. Hamdouni M1, Bourkadi JE, Benamor J, Hassar M, Cherrah Y, Ahid S. Treatment outcomes of drug resistant tuberculosis patients in Morocco: multicentric prospective study. BMC Infectious Diseases 2019, 19:316. https:// doi.org/10.1186/s12879-0193931-5.

80. Munz M, Bergstrom K. A guide for tuberculosis treatment supporter, Stop TB WHO Geneva, Switzerland; WHO/CDS/TB/2002. [Google Scholar]

81. Hussain S, Hasnain J, Hussain Z, Badshah M, Siddique H, _Fiske C, _Pettit A. Type of Treatment Supporters in Successful Completion of Tuberculosis Treatment: A Retrospective Cohort Study in Pakistan. Open Infect Dis J. 2018; 10: 37-

42.doi: $10.2174 / 18742793018$ 10010037).

82. Soomro HM, Qadeer E, Khan MA, Morkve O. Treatment Supporters and Their Impact on Treatment Outcomes in Routine Tuberculosis Program Conditions in Rawalpindi District, Pakistan. Tanaffos. 2012; 11(3): 15-22. PMCID: PMC4153209 PMID: 25191423)

83. Trunz BB, Fine PEM, Dye C. Effect of BCG vaccination on childhood tuberculous meningitis and military tuberculosis worldwide: a meta-analysis and assessment of costeffectiveness. Lancet 2006; 367: 1173-1180 
84. Colditz GA, Brewer TF, Berkey CS, Wilson ME, Burdick E, Fineberg HV, et al. The efficacy of bacillus Calmette-Gue'rin vaccination of newborns and infants in the prevention of tuberculosis: meta-analyses of the published litera-

ture.JAMA1994; 271 (9): 689702

85. Nguipdop-Djomo P, Heldal E, Rodrigues LC, Abubakar I, Mangtani P. Duration of BCG protection against tuberculosis and change in effectiveness with time since vaccination in Norway: are trospective population-based cohort study. Lancet Infect Dis 2016; 16:219-26.
86. Hesseling AC, Cotton MF, Fordham von Reyn C, Graham SM, Gie RP, Hussey GD. Consensus statement on the revised World Health Organization recommendations for $\mathrm{BCG}$ vaccination in HIV-infected infants. Int J Tuberc Lung Dis 2008; 12: 1376-1379.

87. WHO. Essential actions for effective TB infection control: safety without stigma. 2008, Geneva, http://www.stoptb.org/ wg/tb_hiv/assets/documents/ TBHIV\%2O

Infection\%20Control\% 20Fact\%20Sheet.pdf).
88. Jereb J, Etkind SC, Joglar OT, Moore M, Taylor Z. Tuberculosis contact investigations: outcomes in selected areas of the United States, 1999. Int. J. Tuberc. Lung Dis. 2003;7(12) Suppl 3: S384-S390. [PubMed] [Google Scholar]

89. Dodd PJ, Prendergast AJ, Beecroft C, Kampmann B, Seddon JA. The impact of HIV and antiretroviral therapy on TB risk in children: a systematic review and metaanalysis. Thorax 2017; 72: 559-575 